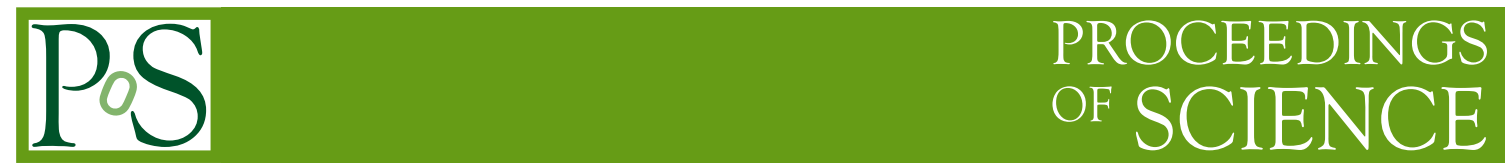

\title{
Cathode Pad Chambers at NICA/MPD
}

\author{
J. Fedorišin*, O.V. Rogachevsky, Y.T. Kiryushin \\ VBLHE, JINR, Dubna \\ E-mail: fedorisin@jinr.ru, rogachevsky@jinr.ru, yukir@sunse.jinr.ru
}

Covering pseudorapidity interval $2<|\eta|<3$, the Cathode Pad Chambers (CPC) are part of the tracking system of the MPD detector. The CPC tracking abilities depend on its geometrical layout which defines the detector occupancy and errors of reconstructed track coordinates. This MC study presents the method for estimating track coordinates along with their experimental errors which are subsequently used in the tracking algorithm in order to determine tracks geometric and kinematic characteristics.

XXI International Baldin Seminar on High Energy Physics Problems September 10-15, 2012

JINR, Dubna, Russia

* Speaker. 


\section{Introduction}

The development of Cathode Strip Chambers (CSC) and Cathode Pad Chambers (CPC) has made a significant progress in recent years. CSC's are usually less expensive, yet on the other hand they provide similar or even better spatial resolution than other types of gas and wire chambers. This resolution typically ranges from 40 to $100 \mu \mathrm{m}$. Another important virtue of CSC's is their fast response that gives them an edge over multiwire drift chambers. Due to lower costs CSC's can be utilized in experiments where high precision large surface detectors are needed which is undoubtedly the case of all the experiments studying ultrarelativistic heavy-ion collisions. These experiments are characterized by large multiplicities of secondary particles which can be therefore distinguished only by means of high resolution detectors.

This article is dedicated to study of the CPC's in the forthcoming experiment NICA/MPD proposed by the Joint Institute for Nuclear Research in Dubna to investigate the properties of extremely dense and hot nuclear matter at the collider energies $\sqrt{s_{N N}}=4-11 \mathrm{GeV}$ [1]. This goal is part of a large-scale international effort devoted to exploration of the phase diagram of strongly interacting QCD matter. While up to now most work within the RHIC and LHC experiments has been done in the region of high temperatures and low net baryon densities, the domain of intermediate temperatures and baryon densities has been only partially scrutinised. This is expected to be accomplished not only in the NICA/MPD experiment but also in the experiments operated by a few other world leading laboratories, namely BNL (STAR and PHENIX at low-energy RHIC) [2],[3], CERN (NA61/SHINE at SPS) [4] and GSI-FAIR (CBM) [5]. Main goal of all the mentioned experiments is to search for possible signals of deconfinement and the phase transitions accompanied by chiral symmetry restoration as well as to locate the QCD critical point [6].

\section{Layout of MPD Cathode Pad Chambers}

The MPD experimental apparatus was designed to meet ambitious physics demands of the proposed NICA/MPD experiment. The apparatus should be able to work in a high track multiplicity environment, to cover a large phase space, to operate at high interaction rates and to identify particles with excellent precision and high efficiency.

MPD experimental layout that suits all these requirements is presented in Fig. 1. The included detector subsystems are elaborately described in [1].

The ion beams pass in opposite direction along $z$ axis and collide at the center of the experimental apparatus at $z=0 \mathrm{~cm}$. The proposed detector composition enables to detect secondary particles with pseudorapidities from the interval $0 \leq|\eta| \leq 3$.

The CPC is tracking detector included in the MPD configuration. Its main role is to provide additional track points between the Inner Tracker (IT) and the TOF in order to mitigate and refine track reconstruction in pseudorapidity interval $2<|\eta|<3$.

There are altogether four CPC's placed at $z= \pm 172,5 \mathrm{~cm}$ and $\pm 204,5 \mathrm{~cm}$, i.e. they are positioned along $z$ axis and arranged in pairs symmetrically with respect to the origin of the coordinate system, as shown in Fig. 1.

Schematic draft of the single CPC is shown in Fig. 2. As for the geometric proportions, the CPC shape is in fact more similar to disc or pancake because its radial size obviously dominates the 


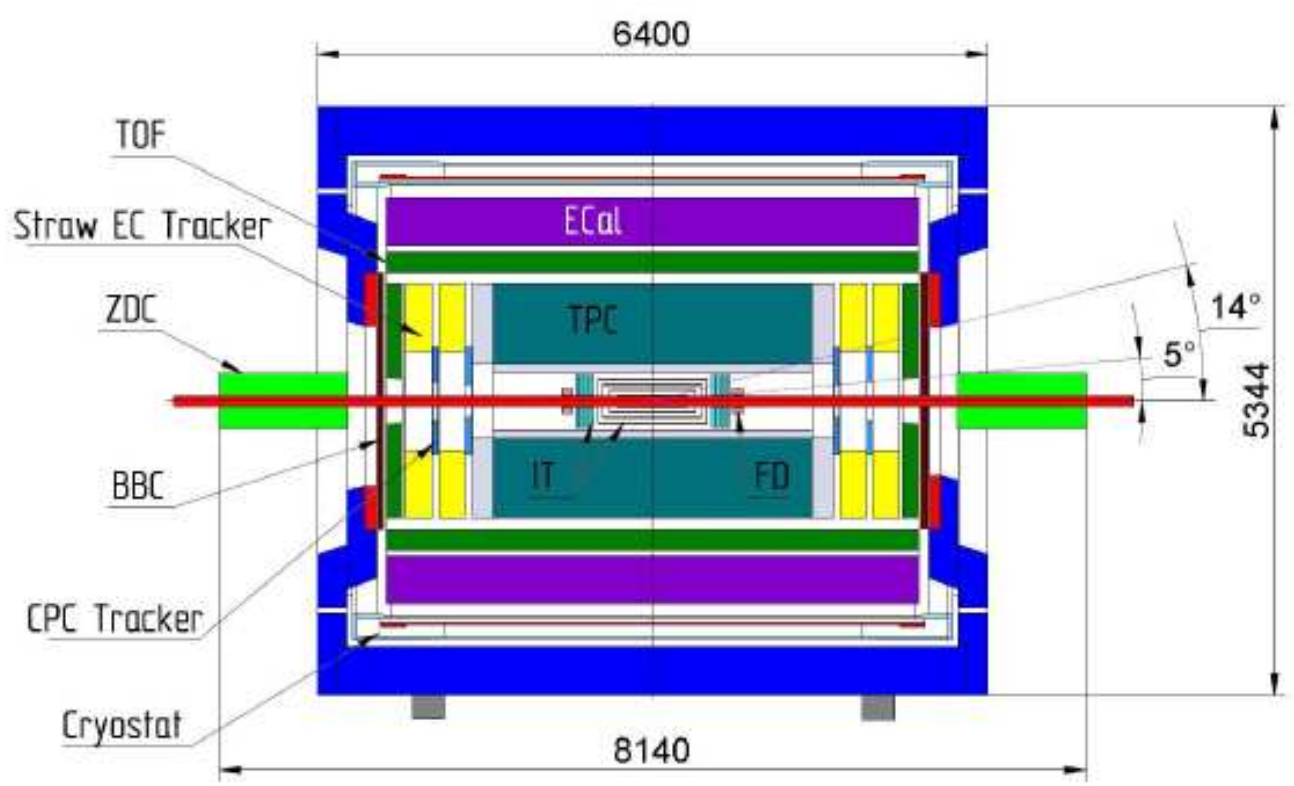

Figure 1: Cutaway side view of the central MPD with basic dimensions expressed in milimeters.

longitudinal one. CPC consists of nine segments - five module panels and four active gas volumes. The working gas which is a $80 \% / 20 \%$ mixture of $\mathrm{Ar}$ and $\mathrm{CO}_{2}$ is sealed in $0.5 \mathrm{~cm}$ wide gap between two neighbouring module panels.

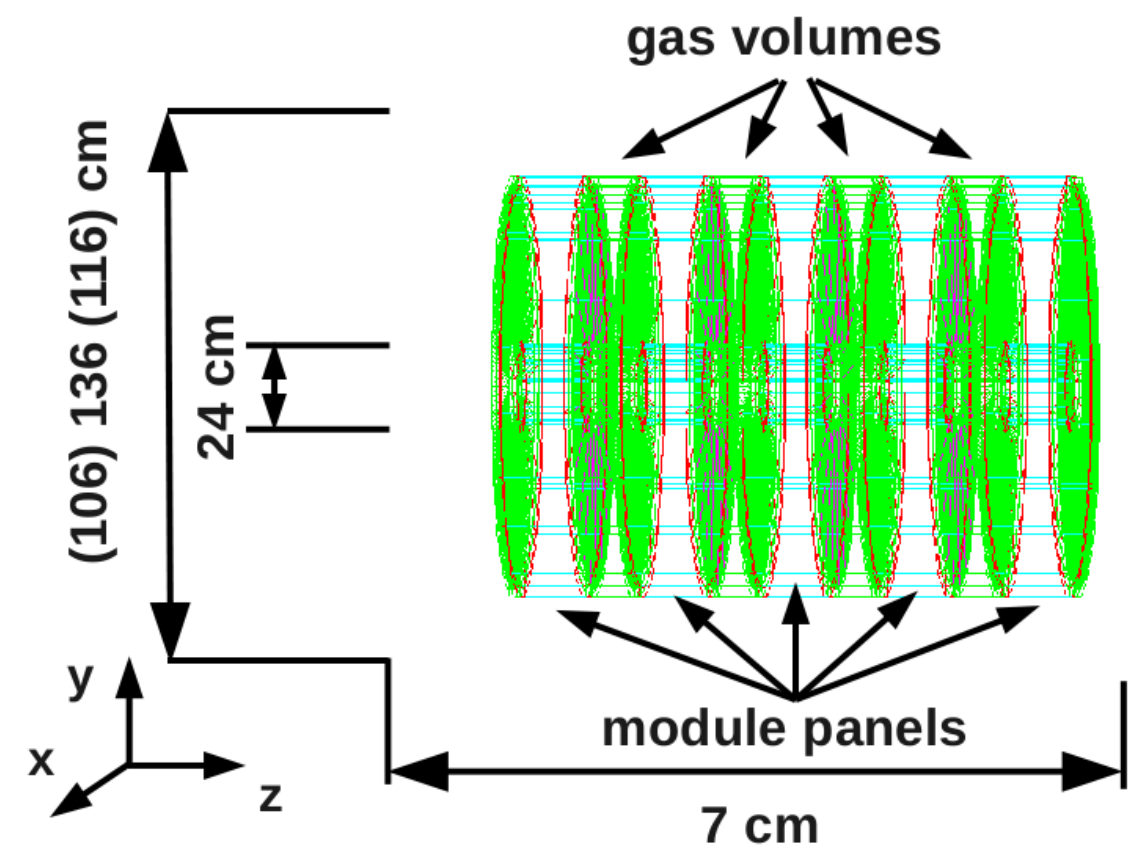

Figure 2: CP chamber schematic outline. The values in parentheses are CPC outer radii in the alternative CPC geometries. 
The module panel has more complex structure which includes a few layers mostly of plastic or polymeric materials such as Rohacell, carbon fiber composite (CFC) and G10. The panel structure as well as the proportions of the used materials are indicated in Fig. 3.

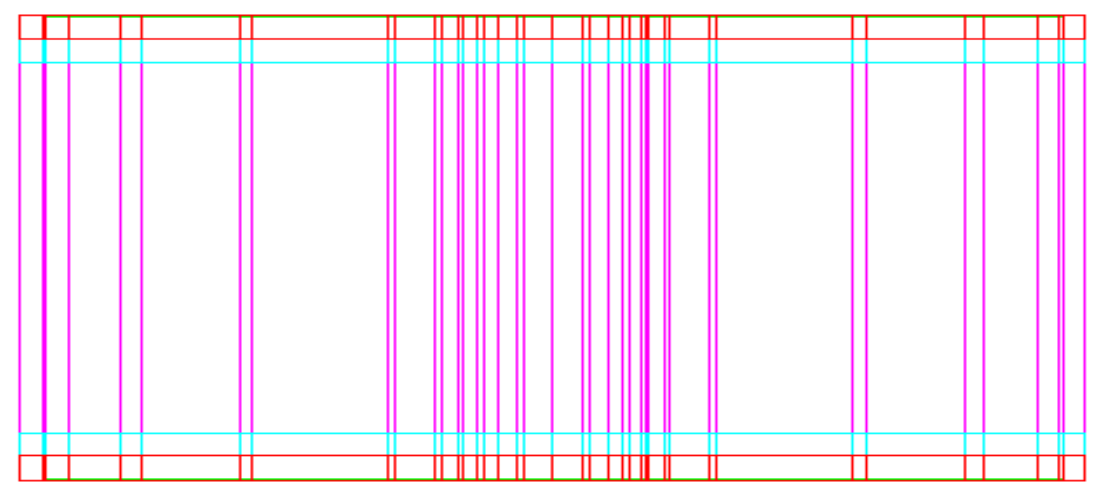

Figure 3: CPC module panel structure. Layers of Rohacell, CFC, G10 and copper are represented by magenta, blue, red and green colours respectively.

Most of panel volume is formed by thick layer of Rohacell which is sandwiched by two thin layers of CFC and G10. Total width of the CPC panel is $1 \mathrm{~cm}$.

G10 is composite material, basically a glass-reinforced epoxy laminate. G10 is also known as non-self-extinguishing predecessor of FR4. Carbon fibre composite is carbon-fiber-reinforced polymer or carbon-fiber-reinforced plastic, extremely strong and light. The polymer is most often epoxy, but other polymers, such as polyester, vinyl ester or nylon, are sometimes used. Apart from carbon fibers, the composite may contain also other fibers, such as Kevlar, aluminium, or glass fibers. ROHACELL is a foam-like rigid expanded plastic material for lightweight sandwich constructions. It has excellent mechanical properties, high dimensional stability under heat, solvent resistance and, particularly at low temperature, a low thermal conductivity. The strength and moduli values are the highest for any foamed plastic in its density range.

Each CPC module panel is coated on both sides by elaborate structure of hundreds of copper pads (strips). They are only $18 \mu \mathrm{m}$ thick which make them barely visible in Fig. 3. Modern photo-etching methods are capable to place the copper strips with high accuracy even on surfaces of several $\mathrm{m}^{2}$ which is crucial for precise determination of coordinates of passing tracks. The structure and arrangement of cathode strips depend on which coordinate is to be measured by the given cathode plane. The MPD CPC's are designed to measure polar track coordinates since polar or cylindrical coordinate systems are more comfortable and more natural for HEP experiments than the Cartesian one.

Strip layouts for the radial and the azimuthal cathode planes are shown in Fig. 2.

The azimuthal cathode plane in Fig. 2 (left) is more structured along azimuthal angle where it is divided to 512 (or 384) ${ }^{1}$ azimuthal strips in the inner radial sector and 1012 ( or 768) strips in

\footnotetext{
${ }^{1}$ The values in parentheses are used in the newer CPC geometry.
} 

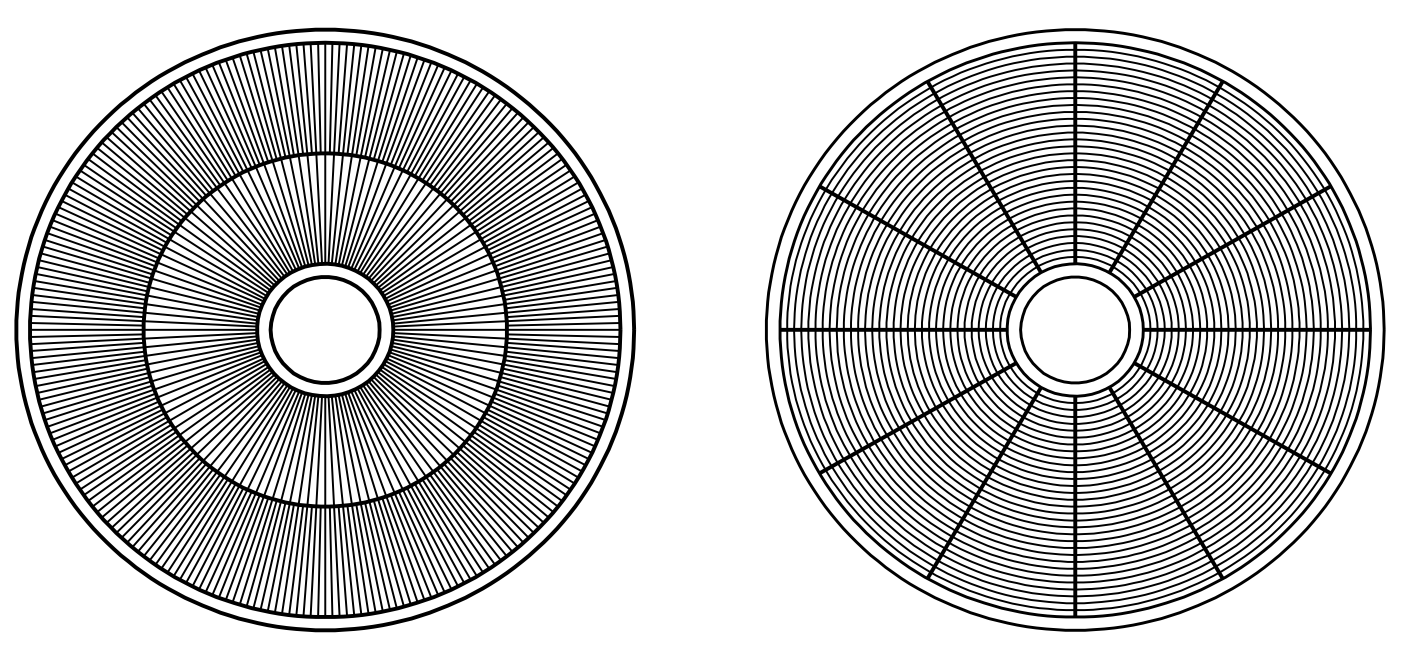

Figure 4: Layout of CPC cathode pad structure in $\varphi$ coordinate (left) and $R$ coordinate (right).

the outer one. The total number of readout channels is thus: $512+512 \times 2=1536$ in the old CPC geometry or 1152 in the new one.

On the other hand, the structure of the cathode plane in Fig. 2 (right) implies it is designed to measure radial coordinate. The radial plane is divided into 12 azimuthal sectors, each one comprising 128 (or 96) radial strips. The total number of readout channels in this plane is then $12 \times 128=1536$ (or 1152 in the new geometry).

\section{Main goal of the simulation studies}

When designing a new detector, it is always helpful to have substantiated estimates of its efficiency and capability to perform tasks for which the detector is intended. Such estimates can be obtained by MC simulations which are able to simulate physical experiment as well as the subsequent detector response. Detector response in general depends on its geometric configuration and material composition. Our goal is to estimate optimal geometric characteristics and material composition of the CPC, i.e. to estimate such geometry and other detector properties for which we obtain the best track reconstruction results, namely the track reconstruction efficiency as well as the geometric and kinematic track parameters. For this reason, several versions of CPC geometry have been tested, as already mentioned a few times before.

To achieve these goals, the standard HEP and NICA/MPD software is used in MC simulations: C++, GEANT3, FAIRSOFT, MPDROOT, UrQMD model. These standard programming packages are supplemented by the original procedures reflecting a specific nature of the solved problems.

\section{Working principle of CPC}

Cathode strip/pad chambers are basically multiwire gas chambers with segmented cathodes. When a charged particle passes through the active gas volume of the detector, it produces ionization 
(electron-ion pairs) along its trajectory. These primary electrons drift in the high voltage electric field towards the nearest anode wire. When electrons approach the anode wire, their kinetic energy gradually increases due to an acceleration in the stronger electric field. After reaching the ionization energy of the gas molecules, the electrons knock out further electrons which leads to creation of avalanche in the vicinity of the anode wire. The resulting ion cloud induces charge signal on the cathode strips close to the avalanche location by capacitive coupling.

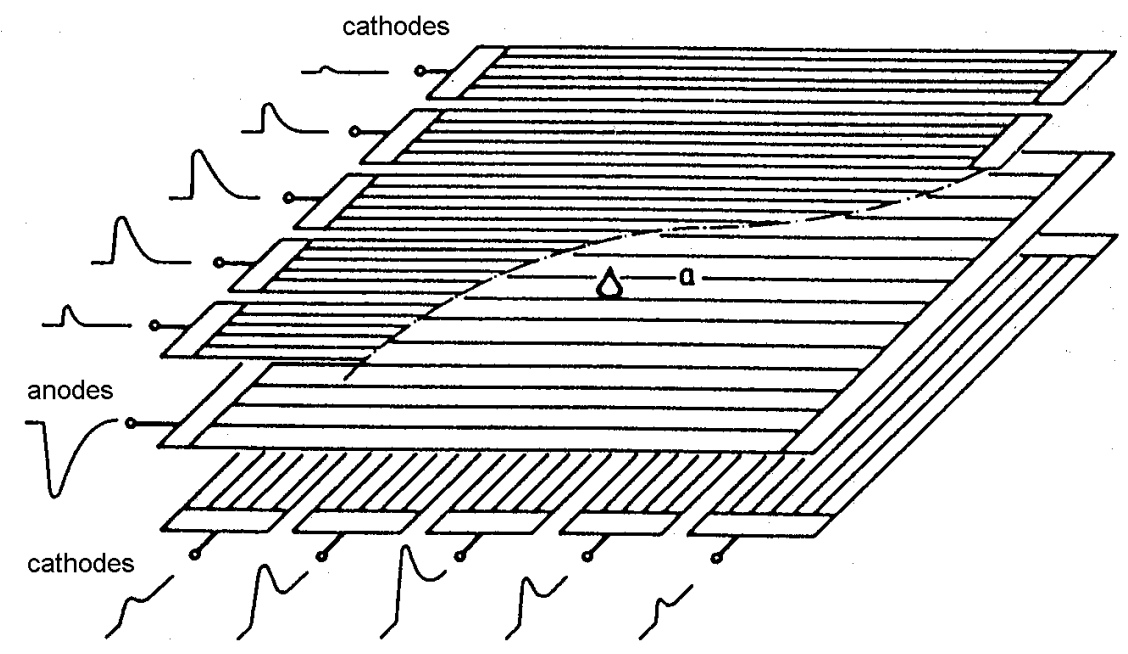

Figure 5: CPC working principle [7]

The working principle of CPC/CSC is in Fig. 5. It is shown for a chamber designed to measure Cartesian coordinates but the chambers measuring polar coordinates work in analogical way. The picture 5 shows anode-wire plane sandwiched by two cathode planes whose strips are mutually rotated by 90 degrees in order to measure both $x$ and $y$ coordinates. The coordinates of passing particle are then estimated from the signals induced on cathode strips by some appropriate position finding algorithm. The methods commonly used for coordinates estimation are presented and discussed at length for instance in [8].

\section{Detector occupancy}

Before proceeding to the estimation of track coordinates with the MPD CPC, let us turn our attention briefly to the estimation of the detector occupancy since this is a quite important detector characteristic and it can be evaluated very fast by the elementary calculations. Providing that each track produces signal only on one cathode strip, the overall mean detector occupancy $\xi$ is defined as

$$
\xi=\frac{N_{\text {hits }}}{N_{\text {total }}}
$$

where $N_{\text {hits }}$ is number of the strips with hits and $N_{\text {total }}$ is number of all strips. The occupancy $\xi$ is usually averaged over a large event sample. In our case, when employing as simulated data sample 500 minbiased $9 \mathrm{GeV}$ AuAu events generated by UrQMD, we obtain $\xi \approx 3 \%$ for both the radial and the azimuthal cathode planes. 
However, since the occupancy $\xi$ is only average value, its informative value is rather limited. Even a low value of $\xi$ does not necessarily mean, there are no overloaded strips, i.e. the strips with hits from two or even more tracks. A presence of such strips is regarded as highly undesired effect because such tracks are experimentally indistinguishable.

In order to estimate the proportion of overloaded strips, the strip occupancy must be studied event-by-event. Its distributions for both the radial and the azimuthal cathode planes are presented in Fig. 6. The simulated data sample is the same as was used previously.
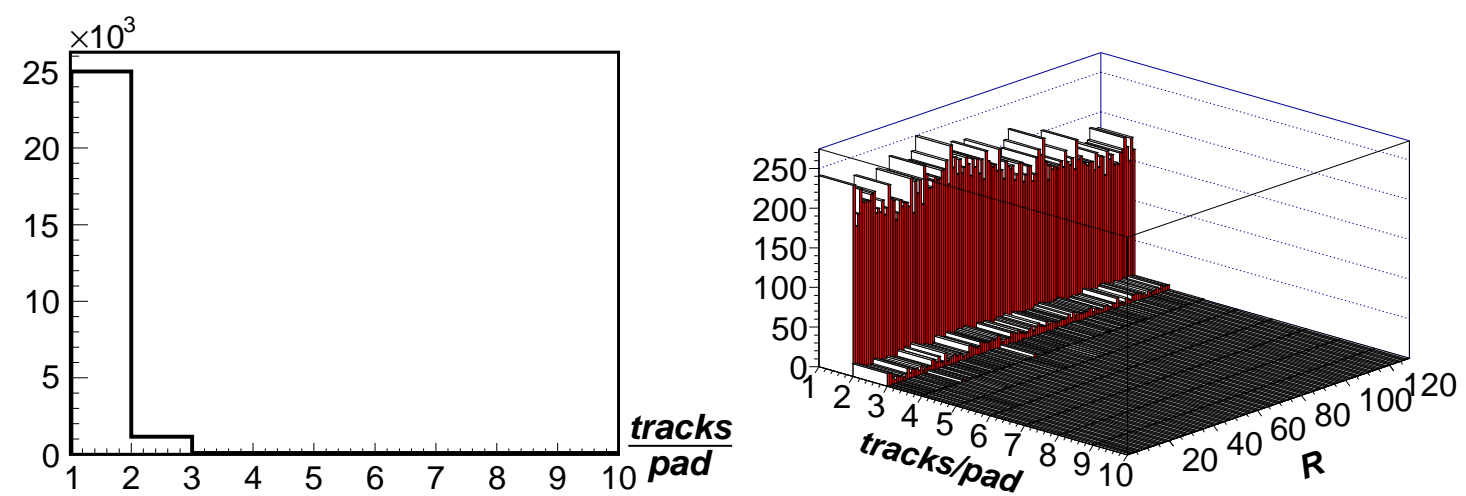

Figure 6: Event-by-event strip occupancy for the azimuthal (left Fig.) and radial (right Fig.) cathode pad planes. The occupancy in right Fig. is shown as function of radius.

The distributions imply that the proportion of overloaded strips is about $4 \%$ of all the strips with hits. The overloaded strips indicate a presence of very close tracks. More thorough investigations reveal that the pairs of very close tracks are above all secondary particle tracks ( $\delta$-electrons) produced in the inactive volumes of the detector.

Before proceeding to the track coordinates estimation, we must adopt more refined approach based on the more precise modeling of the measured signals and on the sophisticated methods of signal analysis.

\section{Distribution of cathode charge}

In fact, the cathode charge induced by passing particle is observed not only in the nearest strip but in a few neighbouring strips as well. Its distribution in cathode plane is described by two-dimensional Gaussian-like function centered at a true hit position. Thus virtually a whole charge is collected only from the strips within a circular area. Hit position in CPC $r(\varphi)$-plane is subsequently estimated by some suitable method that uses as input cathode charges measured in the strips, providing the positions of strips are well defined.

There are a few parameterizations that relatively precisely decribe cathode charge distribution, for example Gaussian, Lorentzian or hyperbolic secant squared, however, the most accurate description of experimental data is provided by empirical Mathieson formula [9], [10], [11] depending on parameters $K_{1}, K_{2}$ and $K_{3}$ :

$$
\frac{\rho(\lambda)}{q_{a}}=K_{1} \frac{1-\tanh ^{2}\left(K_{2} \lambda\right)}{1+K_{3} \tanh ^{2}\left(K_{2} \lambda\right)}
$$


where

$$
K_{2}=\frac{\pi}{2}\left(1-\frac{\sqrt{K_{3}}}{2}\right), \quad K_{1}=\frac{K_{2} \sqrt{K_{3}}}{4 \arctan \sqrt{K_{3}}}
$$

and $K_{3}=K_{3}\left(r_{a} / s, h / s\right)$, in which $h$ is the anode-cathode separation, $s$ is the anode wire pitch, $r_{a}$ is the anode wire radius and $q_{a}$ is the net anode charge. Hence in this representation the shape of cathode charge distribution is determined only by one parameter $K_{3}$ which is an empirical function of the geometric parameters of CPC [10]. Space coordinate $x$ is expressed through the dimensionless quantity $\lambda=x / h$. When working with polar coordinates, $x$ stands either for radius $R$ or for azimuthal arc $R \Delta \varphi$, assumming the arc length is negligibly small when compared with the full perimeter $2 \pi R$. The Mathieson function calculated with the geometric parameters of MPD cathode pad plane is shown in Fig. 7.

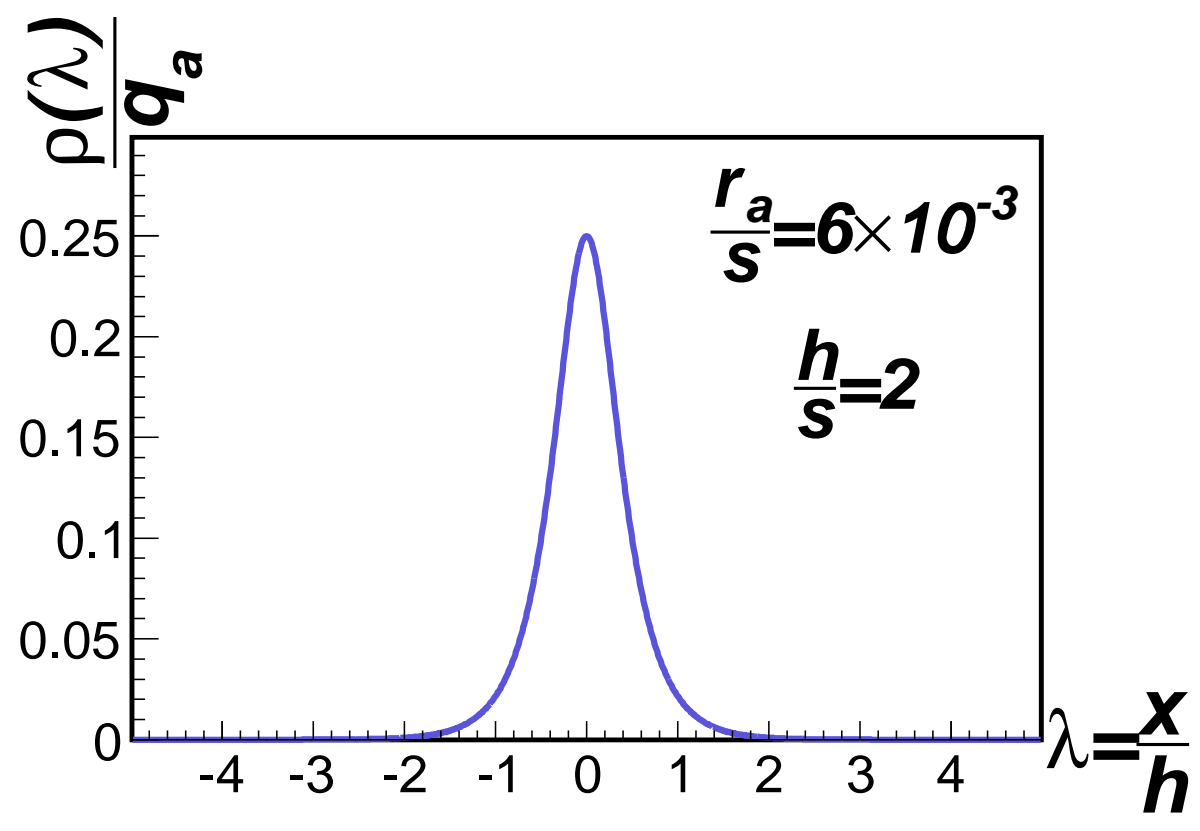

Figure 7: Mathieson function for MPD cathode pad plane

If strips in the opposite cathode planes are mutually perpendicular, two-dimensional Mathieson function can be expressed as the product of two one-dimensional Mathieson functions for the separate coordinates.

Mathieson function describes a shape of cathode charge distributions but in simulated data we need to predict the amplitudes of these distributions as well. It is justified to assume that the magnitudes of cathode charges will copy the distribution of initial charges produced in the ionization of CPC gas. This initial charges spectrum will likewise copy the distribution of energy losses in the detector. On the whole, the spectrum of amplitudes of cathode charges can be approximated by the corresponding spectrum of energy losses. It is well-known that the energy losses of particles are described by Bethe-Bloch formula and their fluctuations are described by Landau (or Vavilov) distribution. The energy losses are in general function of particle momenta $p$ and particle types.

However, in our MC simulations we do not calculate the energy losses spectra ourselves, but instead we employ the spectra $\mathrm{d} E / \mathrm{d} x$ vs $p$ for the needed particle types simulated by GEANT. The 
amplitudes of cathode charges are then generated according to these spectra.

Fig. 8 shows the example of cathode charge distribution in the chosen azimuthal sector of radial pad plane. The peaks (clusters) correspond to the hits of detected tracks.

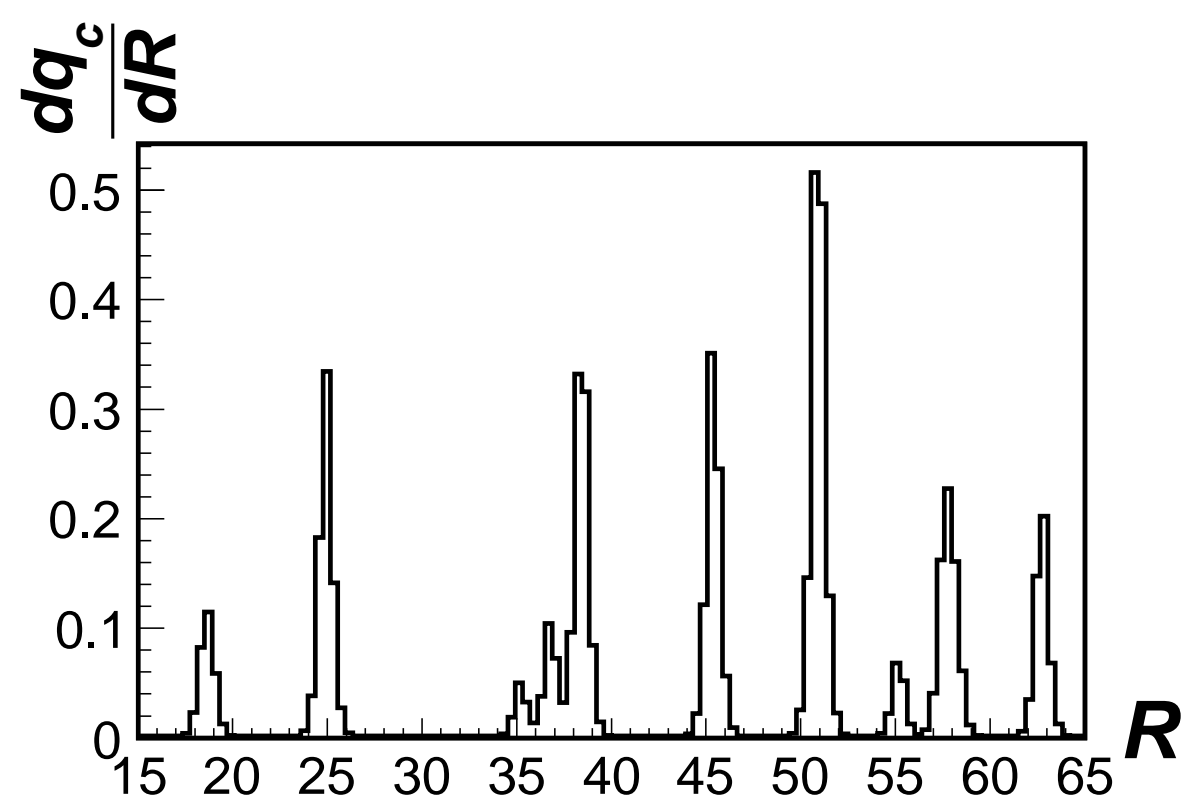

Figure 8: Distribution of cathode charge in the chosen azimuthal sector of radial pad plane. Peaks in the spectrum correspond to the hits of passing tracks.

As we can see, the cathode charges amplitudes may differ significantly, even by the order of magnitude. Furthermore, when charge clusters overlap with each other, their separation becomes difficult, if not quite impossible.

\section{Hit coordinates estimation}

In this section we present the analytical non-iterative method that evaluates hit position from the charges measured in strips around the impact point. Since the proposed method provides a precise analytical solution, no systematic corrections typical for the other position finding techniques [8] are needed.

First, the simple maximum finding algorithm is applied to the cathode charge spectra to find and to separate the peaks if they do not overlap or if they overlap only partially. Then an area of each peak is regarded as integral of Mathieson function. The definite integral of Mathieson function is:

$$
\int_{\alpha}^{\beta} \frac{\rho(\lambda)}{q_{a}} \mathrm{~d} \lambda=D \arctan \left(\frac{1}{A \exp \left(x_{0}\right)+B \exp \left(-x_{0}\right)+C}\right),
$$

where parameters $A, B, C, D$ are functions of the detector geometry-based constants $K_{1}, K_{2}, K_{3}$ and of the integration limits $\alpha, \beta$. The variable $x_{0}$ is center of Mathieson distribution. The integration over one or a few strips gives cathode charge $Q_{c}$ which is a quantity measured in the experiment. The unknown variable is $x_{0}$, coinciding with the true hit position. This variable is evaluated by solving the above equation for $x_{0}$. 
Equation 7.1 can be transformed to the quadratic equation that gives for $x_{0}$ ambiguous solution, in detail presented in Appendix A. In order to decide which solution is correct, Equation 7.1 must be solved for at least two strips. The solutions which coincide in both or more strips are regarded as correct while the differing solutions are discarded.

The presented method provides accurate hit positions, but only for non-overlapping hits. If two or more hits overlap, the accuracy more or less deteriorates, depending on the efficiency of the hit separation algorithm. If the hits overlap to such extent that they become inseparable from each other, then only one average coordinate is estimated for all of them. Moreover, the shape of overlapping cathode charges will differ from the shape of Mathieson function, which will lead to additional error of the estimated coordinate. Another errors arise due to the boundary effects, when the charge clusters produced on the boundaries of radial or azimuthal sectors are incomplete and require a special treatment.

The above-described errors have systematic character. However, the most significant source of errors is electronic noise produced by the amplification system. This statistical noise cannot be fully removed from experimental data and therefore constitutes the main resolution limiting factor. In the simulated data this noise must be artificially generated and added to the cathode charges spectra before they are analyzed. The electronic noise is in our MC data mimicked by Gaussian white noise with $\sigma=0.5 \%$ of the mean total cluster charge, as recommended in the literature [8], [12].

Fig. 9 shows the empirical resolutions of radius and azimuthal angle achieved for the proposed hit position finding method. The resolution of azimuthal angle is multiplied by radius in order to be expressed in units of length. Then we obtain virtually same resolutions for both the coordinates. The value of $50 \mu \mathrm{m}$ is regarded as very good coordinate resolution for CPC/CSC [8]. There is also undesired systematic shift of a few microns observed in both the presented distributions.
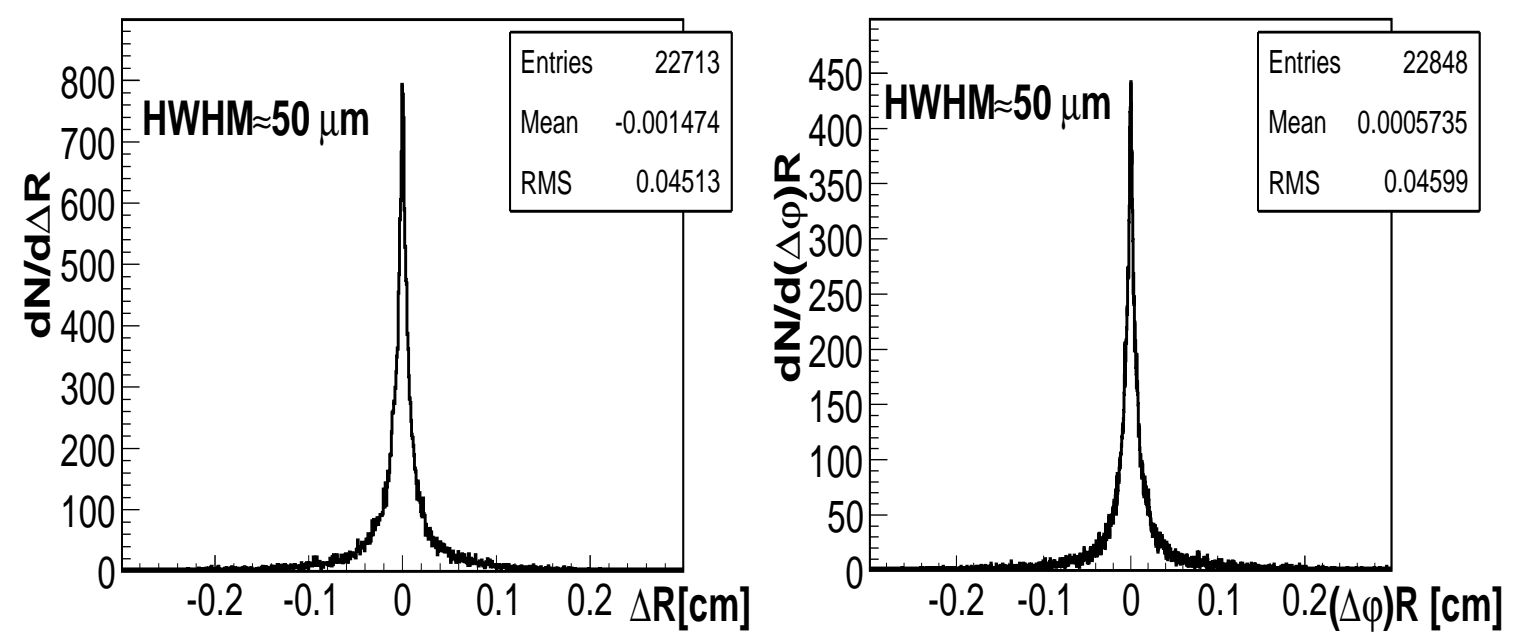

Figure 9: Difference of real and reconstructed radius $\left(R_{\text {rec }}-R_{\text {real }}\right)$ (left) and difference of real and reconstructed azimuth $\left(\varphi_{\text {rec }}-\varphi_{\text {real }}\right)$ of CPC hits (right). The latter quantity is multiplied by radius $R_{\text {real }}$.

Nevertheless, there exist another possible sources of resolution degradation that may increase the resulting coordinate errors, namely: 
1. cross talk between neighbouring readout channels;

2. calibration uncertainties (offset and nonlinear gain);

3. ADC digitization error;

4. mechanical tolerances in the chamber construction;

5. inclined tracks;

6. influence of strong magnetic fields (Lorentz angle);

7. delta electrons production.

An impact of these effects on the coordinate resolutions of the MPD CPC has not been investigated yet.

On the other hand, we already developed relatively simple method to estimate the coordinate errors in the experimental data where, unlike in the simulated data, the information on true hit coordinates are not available. The method estimates coordinate error as a mean fluctuation of the cathode charges defining the charge cluster.

Estimated coordinates of track hits along with the corresponding errors constitute everything that is needed for the recontruction of particle tracks. Development and testing of the proper tracking algorithm is currently underway.

\section{Summary an outlook}

Here is a short summary of what has been done for the CPC up to now:

- detector geometry (several layouts tested);

- detector occupancy, occupancy of strips;

- simulation of hits, including electronic noise;

- cluster finding;

- estimation of hit coordinates and their errors.

What is underway or ahead?

- tracking;

- investigation of other effects that may have impact on track reconstruction;

- testing of alternative methods included in track reconstruction;

- further improvements, tuning. 
Only after accomplishing all the planned tasks, we gain enough information to thoroughly judge how justified the proposed CPC geometries are. The total track reconstruction efficiency is complex function of the hit finding and reconstruction efficiency, the tracking algorithm efficiency, the CPC geometry configuration and material composition, and last but not least, the properties of the processed track ensembles, such as their multiplicities, space distributions and densities, track curvatures, etc. All the mentioned components are not independent but influence each other in nontrivial ways which are difficult, if not impossible, to assess. However, the MC simulation procedures provide us with a powerfull tool to examine all the facets of the employed track reconstruction algorithm in order to use the acquired information to optimize this algorithm or possibly the entire detector structure.

\section{A. Solutions of the integral of Mathieson function for hit coordinates}

Equation 7.1 can be transformed to quadratic equation using substitution $y=e^{x}$. Roots $y_{1,2}$ of the quadratic equation are well-known:

$$
y_{1,2}=\frac{-b \pm \sqrt{b^{2}-4 a c}}{2 a} .
$$

The parameters $a, c, b$ are defined respectively as follows:

$$
a=1+\frac{\left(K_{3}-1\right)^{2}}{4 K_{3}}, \quad c=\frac{\left(K_{3}+1\right)^{2}}{4 K_{3}} \exp (\alpha+\beta)
$$

and

$$
b=\frac{1-K_{3}^{2}}{4 K_{3}}[\exp (\beta)+\exp (\alpha)]+\frac{K_{3}+1}{2 \sqrt{K_{3}}} \frac{[\exp (\beta)-\exp (\alpha)]}{\tan \left(\frac{-Q_{c} K_{2} \sqrt{K_{3}}}{h * K_{1}}\right)} .
$$

The integrated cathode charge $Q_{c}$ as well as the parameters $K_{1}, K_{2}, K_{3}, \alpha, \beta$ and $h$ are defined and described in sections 6 and 7 .

Hit coordinate is eventually obtained by logarithmization of A.1. The redundant solution must be eliminated by applying additional restrictions.

\section{References}

[1] MPD Collaboration, The MultiPurpose Detector - MPD - to study Heavy Ion Collisions at NICA, (Conceptual Design Report), http://nica.jinr.ru/files/CDR_MPD/MPD_CDR_en.pdf, Dubna, 2008.

[2] Bedangadas Mohanty (for the STAR Collaboration), STAR experiment results from the beam energy scan program at the RHIC, J. Phys. G: Nucl. Part. Phys. 38 (2011) 124023.

[3] Alexander Schmah (for the STAR Collaboration), Highlights of the Beam Energy Scan from STAR, arXiv:1202.2389 [nucl-ex].

[4] Antoni Aduszkiewicz, for the NA61 Collaboration, NA61/SHINE at the CERN SPS: plans, status and first results, Acta Physica Polonica B vol. 43 (2012) 635, arXiv:1201.5879 [nucl-ex].

[5] V. Friese, The CBM Experiment - a Status Report, Physics of Atomic Nuclei, 75, (2012), 585-588. 
[6] B. Friman, C. Höhne, J. Knoll, S. Leupold, J. Randrup, R. Rapp, P. Senger, The CBM Physics Book: Compressed Baryonic Matter in Laboratory Experiments, Lect. Notes Phys. 814, Springer-Verlag Berlin, Heidelberg 2011.

[7] G. Charpak et al., Nucl. Instr. Meth. 148 (1978) 471.

[8] R. Wurzinger, Y. Le Bornec, N. Willis, Monte Carlo studies on Cathode Strip/ Pad Chambers for the ALICE Di-Muon Arm, Report: IPNO-DRE-96-98; DE98-611581.

[9] E. Mathieson and J.S. Gordon, Cathode Charge Distributions In MultiWire Chambers: 2. Approximate and empirical formulae, Nucl. Inst. and Meth. 227,(1984), 277-282.

[10] E. Mathieson, Cathode Charge distribution In Multiwire Chambers: 4. Empirical formula for small anode-cathode separation, NIM in Physics Research, A270 (1988) 602-603.

[11] E. Mathieson and J.S. Gordon, Cathode Charge Distributions In MultiWire Chambers: 1. Measurement and theory, Nucl. Inst. and Meth. 227,(1984), 267-276.

[12] H. Fenker, J. Thomas, M. Brooks, D. Lee, and G. Mills, Precision Interpolating Pad Chambers, Nucl. Inst. and Meth. A 367 (1995) 285-289. 